\title{
STUDY OF THE PHASE TRANSITION DYNAMICS OF THE L TO H TRANSITION
}

by

PECENEO

R.A. MOYER, T.L. RHODES, C.L. RETTIG, E.J. DOYLE, K.H. BURRELL, O S T I J. CUTHBERTSON, R.J. GROEBNER, K.W. KIM, A.W. LEONARD,

R. MAINGI, G.D.PORTER, and J.G. WATKINS

DTIC QUALTTY INSSECTED 2

DISTAISUTION OF THS DOCUMENT IS UMMUTED oh

DECEMBER 1997
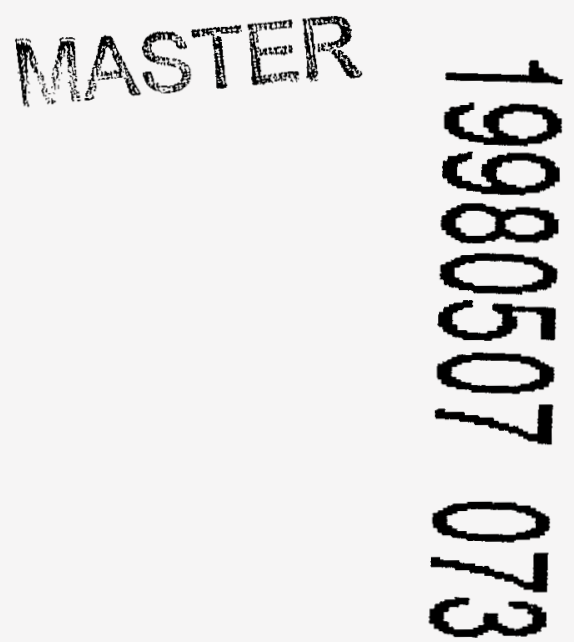


\section{DISCLAIMER}

This report was prepared as an account of work sponsored by an agency of the United States Government. Neither the United States Government nor any agency thereof, nor any of their employees, makes any warranty, express or implied, or assumes any legal liability or responsibility for the accuracy, completeness, or usefulness of any information, apparatus, product, or process disclosed, or represents that its use would not infringe privately owned rights. Reference herein to any specific commercial product, process, or service by trade name, trademark, manufacturer, or otherwise, does not necessarily constitute or imply its endorsement, recommendation, or favoring by the United States Government or any agency thereof. The views and opinions of authors expressed herein do not necessarily state or reflect those of the United States Government or any agency thereof. 


\title{
STUDY OF THE PHASE TRANSITION DYNAMICS OF THE L TO H TRANSITION
}

\author{
by \\ R.A. MOYER, † T.L. RHODES, ‡ C.L. RETTIG, $\ddagger$ E.J. DOYLE, $¥$ K.H. BURRELL, \\ J. CUTHBERTSON, ${ }^{\dagger}$ R.J. GROEBNER, K.W. KIM, $\ddagger$ A.W. LEONARD, \\ R. MAINGI,, G.D.PORTER, $\triangle$ and J.G. WATKINS\#
}

\author{
tUniversity of California, San Diego \\ ‡University of California, Los Angeles \\ Oak Ridge National Laboratory \\ $\Delta$ Lawrence Livermore National Laboratory \\ \#Sandia National Laboratories, Albuquerque
}

This is a preprint of a paper to be presented at the IAEA Technical Committee Meeting on H-mode Physics, September 22-24, 1997, Kloster-Seeon, Germany and to be published in Special Issue of Plasma Physics \& Controlled Fusion.

\author{
Work supported by \\ the U.S. Department of Energy \\ under Contract Nos. DE-AC03-89ER51114, W-7405-ENG-48, \\ DE-AC05-960R22464, DE-AC04-94AL85000, and Grants \\ DE-FG03-95ER54294, DE-FG03-86ER53266
}

GA PROJECT 3466

DECEMBER 1997 


\section{ABSTRACT}

A highly radiating zone (MARFE) just above the divertor $\mathrm{X}$-point has been used to access the marginal transition regime $\mathrm{P}_{\text {sep }} \approx \mathrm{P}_{\text {thres }}$ to study the existence of a critical point for the $\mathrm{L}$ to $\mathrm{H}$ transition. Phase transition models predict that at the critical point, the transition duration increases and the plasma parameters vary continuously between $\mathrm{L}$-mode and $\mathrm{H}$-mode. In these experiments, the $\mathrm{L}$ to $\mathrm{H}$ transition duration increased 50-100 times over fast transitions. However, the evolution of $E_{\mathrm{I}}$ shear, edge density gradient, $\mathrm{H}$-mode pedestal, and fluctuations is essentially unchanged from that in fast transitions. The only difference is in the speed with which and the degree to which the fluctuation amplitudes are transiently reduced. This difference is understandable in terms of the time scales for fluctuation amplitude reduction $(\leq 100 \mu \mathrm{s})$ and edge pressure gradient increase (several $\mathrm{ms}$ ), provided the edge fluctuations are pressuregradient driven. 


\section{INTRODUCTION}

Quantitative understanding of the physics of the $\mathrm{L}$ to $\mathrm{H}$ transition and $\mathrm{H}$-mode confinement remains an important goal of international fusion research due to its relevance to ITER and its intrinsic merit as a basic plasma science problem. Several years of concentrated experimental and theoretical study (see reviews in $[1,2]$ ) have led to the development of a "standard model" for the $\mathrm{L}$ to $\mathrm{H}$ transition based upon the suppression of turbulent transport in the plasma edge via enhanced decorrelation of the turbulent eddies by sheared $\mathrm{E} \times \mathrm{B}$ convection. Today, self-consistent phase transition models [3-6] show the most promise in explaining the experimental data [2,7]. These models predict the existence of a critical point for the $\mathrm{L}$ to $\mathrm{H}$ transition, where the transition duration approaches infinity and the edge conditions evolve continuously from L-mode to $\mathrm{H}$-mode. But operation near this critical point is complicated by sawtooth heat pulses which trigger a fast $\mathrm{L}$ to $\mathrm{H}$ transition. To date, the most complete experimental study of $\mathrm{L}$ to $\mathrm{H}$ transition dynamics has focused on the limit-cycle-like dithering behavior encountered when the power flowing across the separatrix $P_{\text {sep }}$ is marginal for the $\mathrm{L}$ to $\mathrm{H}$ transition $[8,9]$. In the experiments reported here, a highly radiating zone inside the separatrix and just above the divertor $\mathrm{X}$-point (an " $\mathrm{X}$-point" MARFE [10-12]) was established in the L-mode target plasma to allow access to the marginal transition regime by preventing sawtooth heat pulses from triggering a fast $\mathrm{L}$ to $\mathrm{H}$ transition [13]. 


\section{EVOLUTION OF THE RADIAL ELECTRIC FIELD}

These experiments were carried out in lower single null, deuterium neutral beam heated deuterium discharges in DIII-D with a high target (ohmic) plasma density $\overline{\mathrm{n}}=4-4.5 \times 10^{19} \mathrm{~m}^{-3}$, plasma current $\mathrm{I}_{\mathrm{p}}=0.96 \mathrm{MA}$, toroidal magnetic field $\mathrm{B}_{\phi}=1.75$ $\mathrm{T}$, and auxiliary heating power $\mathrm{P}_{\mathrm{inj}}=1.7 \mathrm{MW}$. Changes in the carbon ion temperature, rotation, and pressure gradient were monitored with charge exchange recombination (CER) spectroscopy [14]. From these data, the contributions to the radial electric field $E_{r}$ were evaluated using the lowest order radial force balance equation for a single ion species:

$$
E_{r}=\frac{1}{n_{i} Z_{i} e} \nabla P_{i}-v_{\theta i} B_{\phi}+v_{\phi i} B_{\theta}
$$

where $E_{r}$ is the radial electric field, $n_{i}$ and $Z_{j}$ e are respectively the ion species density and charge, $\nabla \mathrm{P}_{\mathrm{i}}$ is the ion pressure gradient, $v_{\theta i}$ and $\mathrm{v}_{\phi \mathrm{i}}$ are respectively the ion poloidal and toroidal rotation velocities, and $\mathrm{B}_{\phi}$ and $\mathrm{B}_{\theta}$ are respectively the toroidal and poloidal magnetic field.

In previous $\mathrm{L}$ to $\mathrm{H}$ transition studies, we distinguished between "fast" transitions, in which the H-mode $E_{r}$ well developed too fast ( $\leq 1 \mathrm{~ms}$ ) to be resolved by CER spectroscopy, and "slow" transitions, in which the $E_{\mathrm{r}}$ evolution (2-3 ms) could be measured. Since both fast and slow transitions have fast ( $\leq 1-3 \mathrm{~ms})$ recycling changes and very fast fluctuation suppression $(\leq 100 \mu \mathrm{s})$, this distinction is unimportant for this paper, and both types of transition will be referred to in this paper as "fast".

In Fig. 1, the temporal variation of $E_{\mathrm{r}}$ in a very slow $\mathrm{L}$ to $\mathrm{H}$ transition (right) is compared to that in a fast transition (left). In very slow $\mathrm{L}$ to $\mathrm{H}$ transitions (Fig. 1, right) $E_{r}$ and $E_{T}$ shear develop slowly as indicated by an increase in the carbon ion $v \times B$ contribution to $E_{\mathrm{r}}$ inside the separatrix from 2460-2522 ms [Fig. 1(h)]. During this time, the shear in the impurity ion $\nabla \mathrm{P}_{\mathrm{i}}$ contribution to $\mathrm{E}_{\mathrm{r}}$ does not change [Fig. 1(i)], 


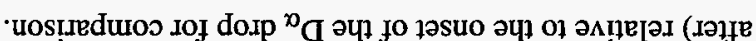

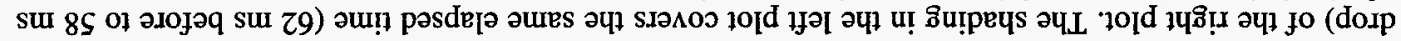

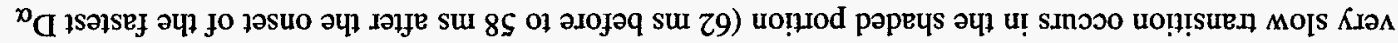

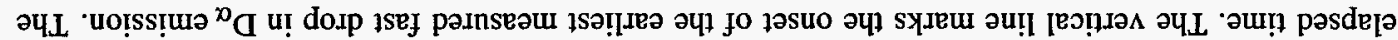

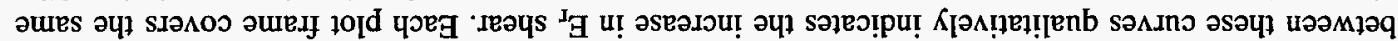

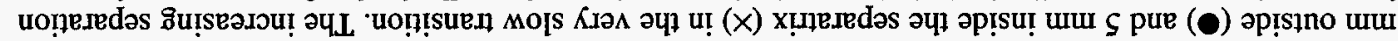

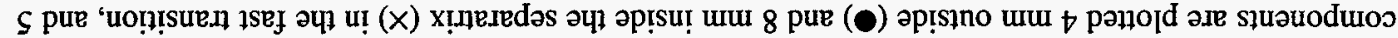

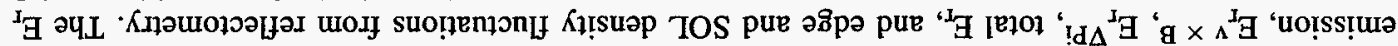

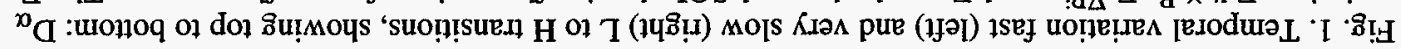

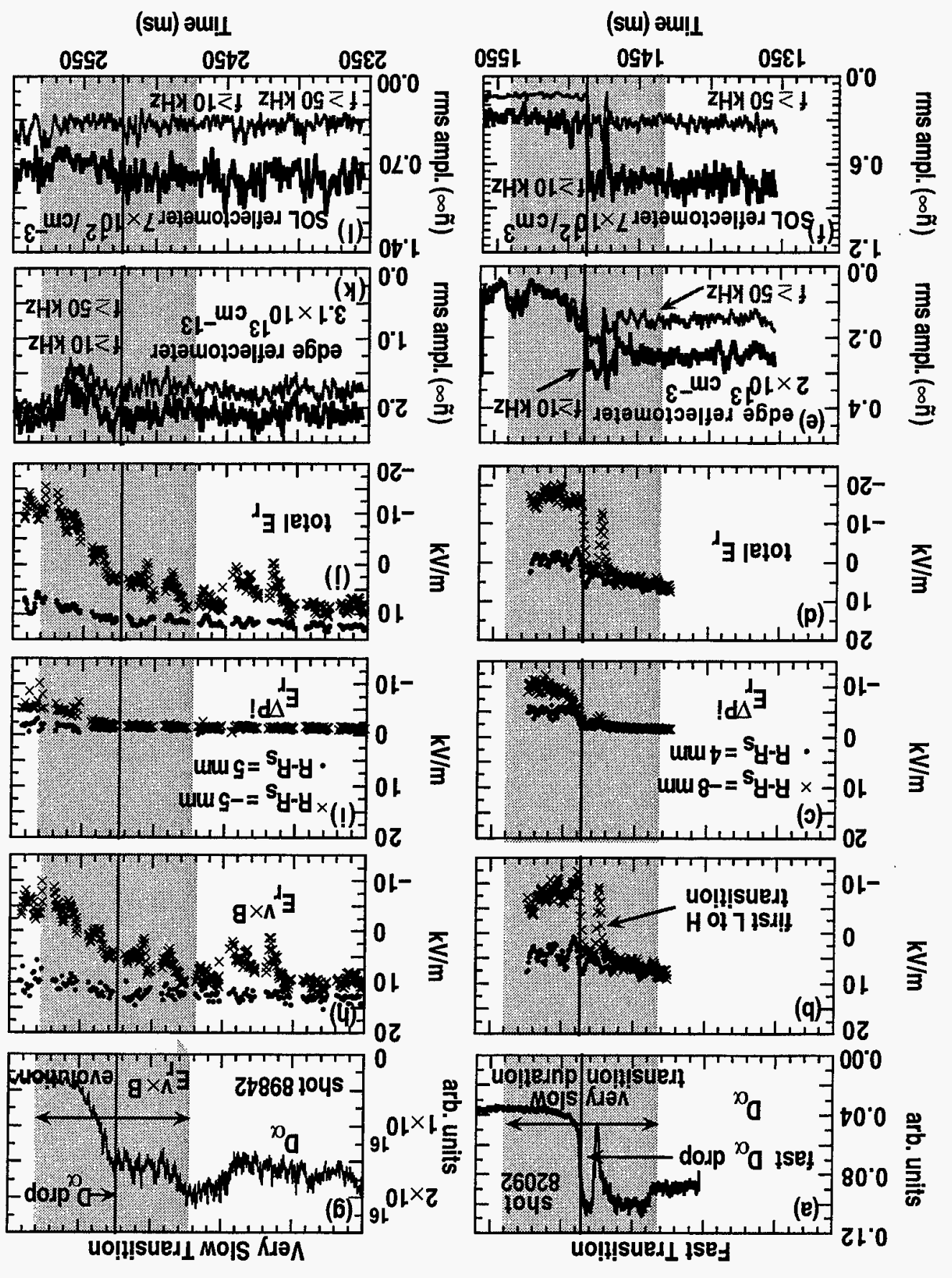


and the $D_{\alpha}$ emission begins to slowly decrease [Fig. 1(g)]. At $2522 \mathrm{~ms}$, the fastest drop in $D_{\alpha}$ begins, and the $E_{r}$ well deepens more rapidly as indicated by a more rapid increase in the shear in $\mathrm{E}_{\mathrm{r}} \mathrm{v} \times \mathrm{B}$. At this time, the shear in $\mathrm{E}_{\mathrm{r}} \nabla \mathrm{Pi}$ begins to increase, but remains small relative to the shear in $E_{r} v \times B$ even after the $D_{\alpha}$ drop is completed at $2600 \mathrm{~ms}$. This sequence is identical to that in fast transitions (Fig. 1, left) aside from the increased time scale, and suggests that even in these cases, the main ion poloidal rotation is the trigger for the transition [7]. However, since the main ion and impurity ion poloidal rotation may be quite different, this inference must still be verified by measurement of the main ion contributions to $E_{r}$ for very slow transitions in helium plasmas where the main ions can be measured directly. 


\section{DEVELOPMENT OF THE EDGE DENSITY GRADIENT AND H-MODE PEDESTAL}

The variation of the edge electron density gradient $\nabla n_{e}$ and the edge electron density pedestal height $\mathbf{n}_{\mathrm{e}}$ ped in fast and very slow $\mathrm{L}$ to $\mathrm{H}$ transitions is shown in Fig. 2. The $\mathrm{H}$-mode pedestal height $\mathrm{n}_{\mathrm{e}}$ ped is the value of ne at the transition from the steep edge gradient region to the relatively flat inner core. These profile parameters are obtained from modified hyperbolic tangent fits to electron density profiles measured by the Thomson scattering system [15]. The hyperbolic tangent consists of a hyperbolic tangent, an offset, and a linear ramp in the plasma core and provides an excellent description of both $\mathrm{L}-$ and $\mathrm{H}$-mode profiles in DIII-D. In both fast and very slow transitions, $\nabla n_{e}$ and $n_{e}$ ped do not change until the onset of the steepest portion of the $D_{\alpha d}$ drop, marked in each plot by the dashed line. In fast transitions, $\nabla \mathrm{n}_{\mathrm{e}}$ jumps to $80 \%$ of the peak ELM-free $H$-mode value within $25 \mathrm{~ms}$ of the onset of the steepest $\mathrm{D}_{\alpha}$ drop. In contrast, $\nabla \mathrm{n}_{\mathrm{e}}$ takes $140 \mathrm{~ms}$ to reach a similar level in the very slow $\mathrm{L}$ to $\mathrm{H}$ transition. After the steepest $D_{\alpha}$ drop begins, $n_{e}{ }^{\text {ped }}$ evolves slowly over $150-200$ ms to the steady state value just before the onset of ELMs in both the fast and very slow transitions.

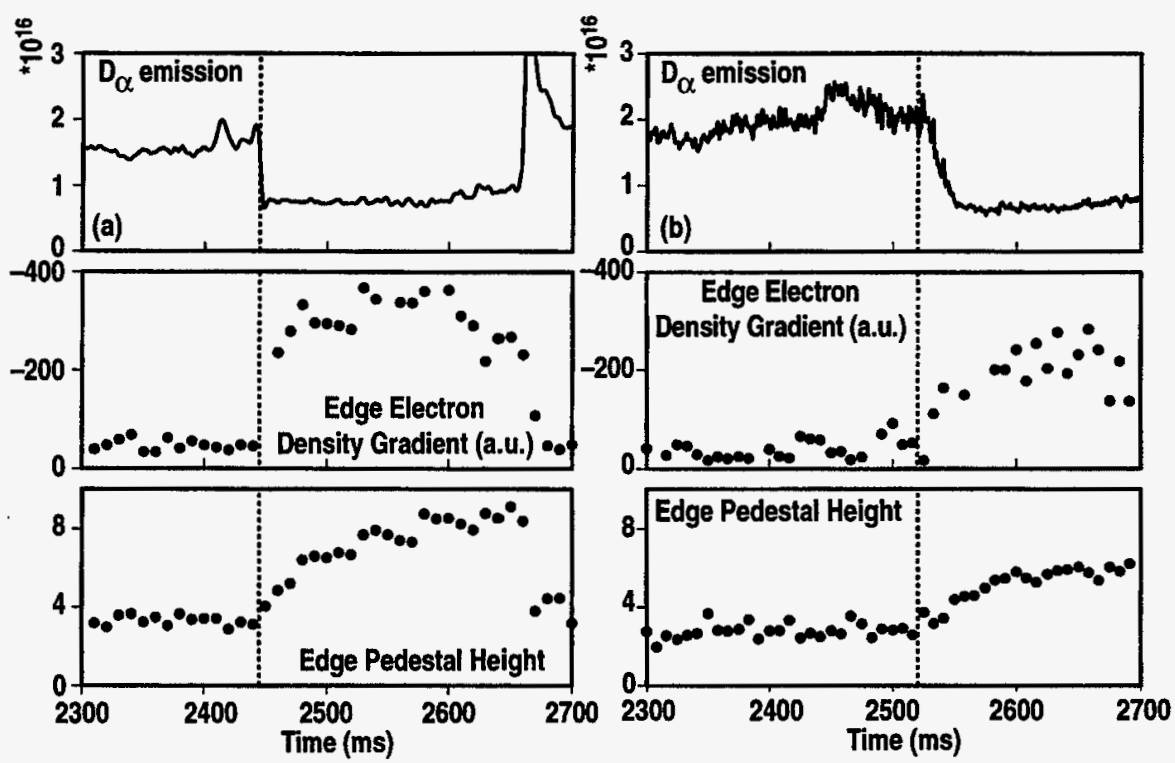

Fig. 2. Temporal variation of (top) $D_{\alpha}$ emission, (middle) edge electron density gradient $\nabla n_{e}$, and (bottom) edge electron density pedestal height $n_{e}{ }^{\text {ped }}$ in (a) fast and (b) very slow $L$ to $H$ transitions. The very slow transition $(b)$ is the same one plotted in Fig. 1. 


\section{SUPPRESSION OF FLUCTUATIONS AND TURBULENT TRANSPORT}

The temporal variation of edge and scrape-off layer (SOL) density fluctuations $\tilde{\mathbf{n}}$ was monitored with reflectometry. During the first stage (2460-2522 ms) of the very slow $\mathrm{L}$ to $\mathrm{H}$ transition (Fig. 1, right), the density fluctuation levels $\tilde{\mathrm{n}}$ don't change in either the edge [Fig. 1(k)] or the SOL [Fig. 1(l)] although the shear in $E_{\mathrm{r}} \mathrm{v} \times \mathrm{B}$ increases [Fig. 1(h)] and the recycling decreases [Fig. 1(g)]. From Fig. 2(b), $\nabla n_{e}$ and $n_{e}{ }^{\text {ped also }}$ do not increase during this time. This behavior is similar to the first stage (2-3 ms before the fast $D_{\alpha}$ drop) of fast $L$ to $H$ transitions [Fig. 2(a)] and (Fig. 1, left) [7]. At $2522 \mathrm{~ms}$ in the very slow transition, the recycling decreases more rapidly and the edge density gradient begins to rise [Fig. 2(b)]. As the density profile steepens, the reflection layer for each reflectometer channel moves outward in radius. Consequently, the temporal variation in the reflectometer powers includes both temporal and spatial variations in the $\tilde{n}$ levels. The edge channel decreases a modest amount over 30-50 ms as the reflection layer moves from the plasma interior into the steep edge gradient region [Fig. 1(k)]. In the SOL, a similarly small reduction in $\tilde{\mathrm{n}}$ is seen [Fig. 1(l)]. This differs from fast $L$ to $H$ transitions only in the speed with which the $\tilde{n}$ levels respond, and in the overall reduction in $\tilde{\mathrm{n}}$ [7].

The $\tilde{\mathrm{n}}$ fluctuation power $\left(\propto\right.$ to $\tilde{\mathrm{n}}^{2}$ ) measured by FIR collective scattering in fast and very slow transitions is compared in Fig. 3. Since the power spectra for these measurements is dominated by the Doppler shift from the $\mathrm{E} \times \mathrm{B}$ rotation, the power in negative frequencies corresponds to fluctuations inside the negative $\mathrm{E}_{\mathrm{r}}$ well, while the power in positive frequencies corresponds to fluctuations deeper in the plasma interior where $E_{r}>0$ [16]. In the fast transition [Fig. 3(a)], the total power in the density fluctuations increases during the $L$-mode period up to the time of the fast $D_{\alpha}$ drop. At $1520 \mathrm{~ms}$, there is a fast $50 \%$ reduction in the density fluctuation power inside the negative $E_{\mathbf{r}}$ well. The fluctuations in the plasma interior increase momentarily before 

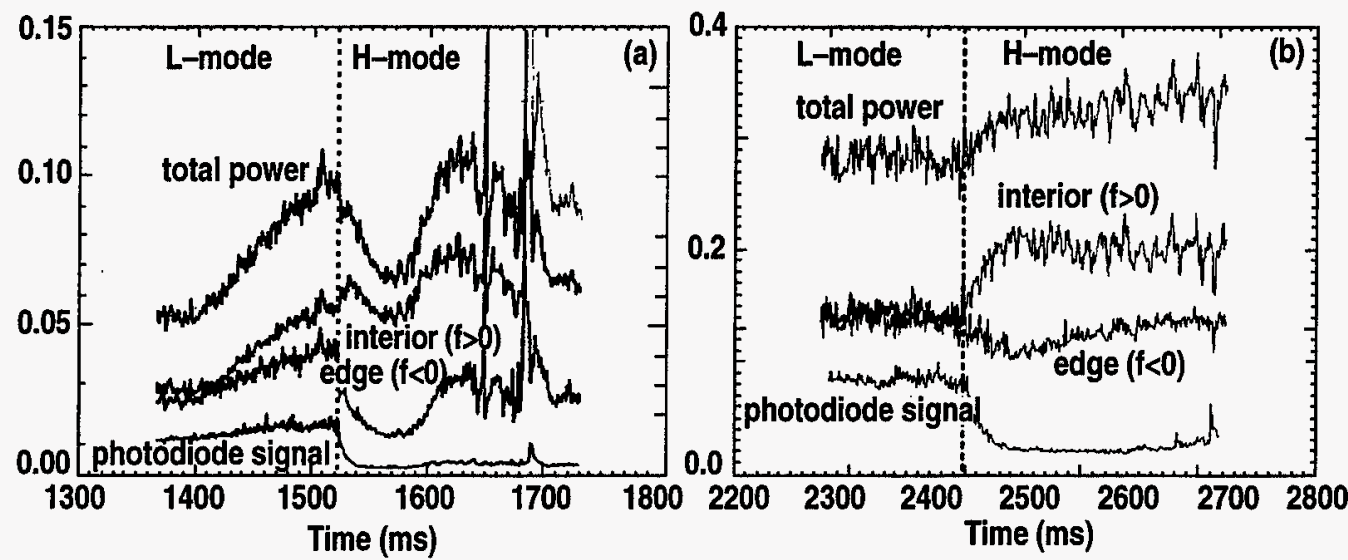

Fig. 3. Evolution of the density fluctuation power $\propto \tan ^{2}$ in (a) fast and (b) very slow $L$ to $H$ transitions as measured by FIR collective scattering. The transition time, taken as the onset of the fastest measured drop in $\mathrm{D}_{\alpha}$, is indicated by the dashed vertical line in each plot. Shown are, from top to bottom, the total power (all frequencies), the power from the interior $(f>0)$, the power from the edge (inside the $E_{r}$ well, $f$ $<0$ ), and the $D_{\alpha}$ emission.

decreasing several tens of milliseconds after the transition [17]. Just before ELMs begin at $1680 \mathrm{~ms}$, the density fluctuations have recovered to nearly the L-mode levels in both the edge and core. In the very slow transition [Fig. 3(b)], the power in the density fluctuations inside the $\mathrm{E}_{\mathrm{r}}$ well slowly decreases $30 \%$ over $40 \mathrm{~ms}$ after the onset of the $\mathrm{D}_{\alpha}$ drop, consistent with the edge reflectometry results [Fig. 1(k)]. The fluctuations later recover to L-mode levels. In the plasma interior, the density fluctuations rise slowly throughout the $D_{\alpha}$ drop while the edge density gradient is steepening [Fig. 2(b)].

The reflectometry and FIR scattering results show that the initial $\mathrm{L}$-mode and final $\mathrm{H}$-mode states are similar for fast and very slow $\mathrm{L}$ to $\mathrm{H}$ transitions. Only the duration of the transition, and the size of the absolute fluctuation amplitude reduction during the transition is different. Reciprocating Langmuir probe measurements taken in the $\mathrm{L}-$ and $\mathrm{H}$-mode phases of very slow transitions confirm that after the $\mathrm{D}_{\alpha}$ drop, density fluctuation levels inside the $\mathrm{E}_{\mathrm{T}}$ shear layer equal or slightly exceed L-mode levels. To within discharge reproducibility, the floating potential fluctuations in $\mathrm{H}$-mode are also equal to L-mode levels. Although the rms amplitudes of the fluctuations in the established $\mathrm{H}$-mode are similar to the $\mathrm{L}$-mode values, the fluctuation-induced particle flux $\Gamma$, equal to the integral of the spectrally resolved particle flux $\Gamma(\omega)$ over the frequency spectrum (Fig. 4), is reduced inside the $E_{\mathbf{r}}$ well by a factor of about 3 . 


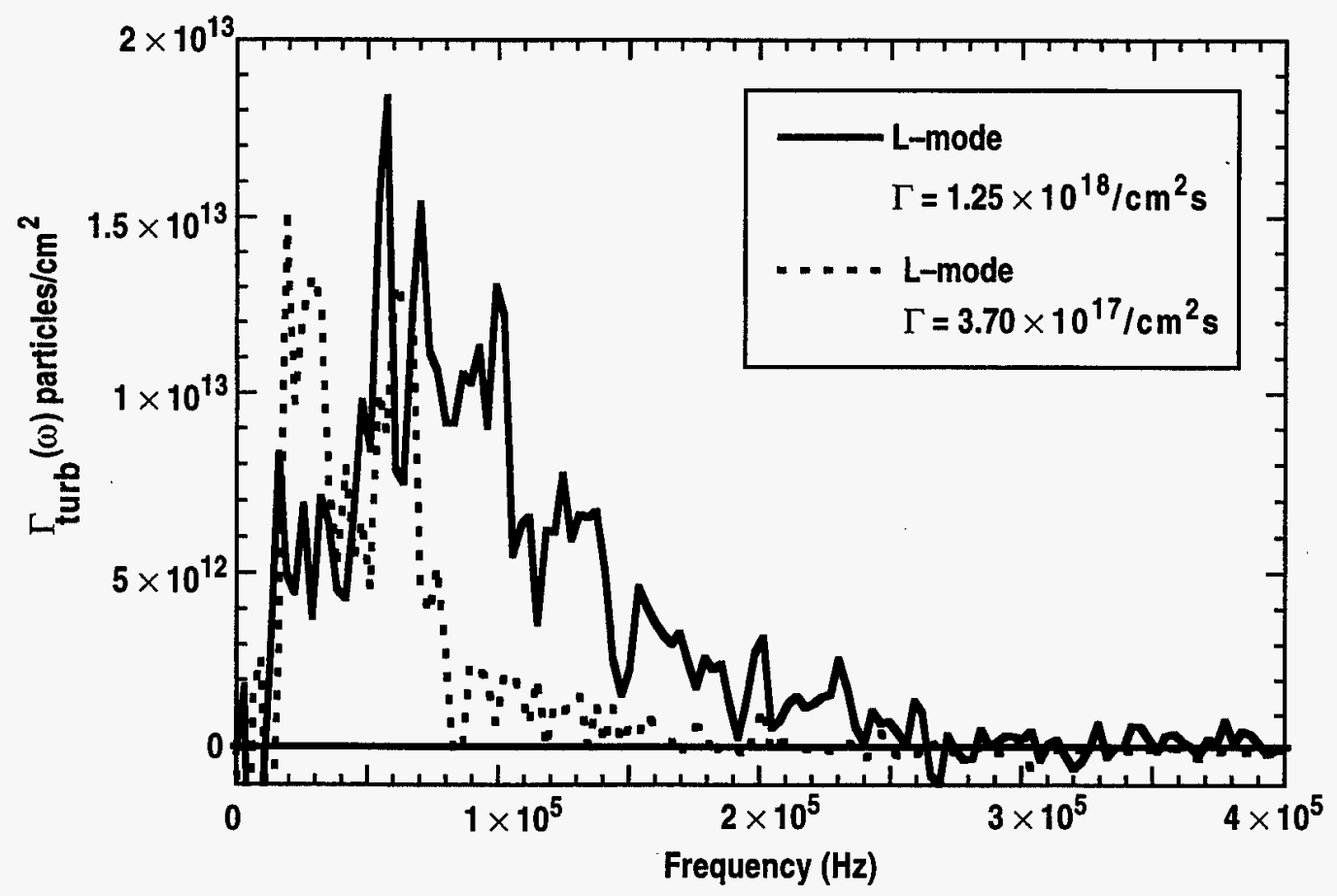

Fig. 4. Comparison of the frequency-resolve turbulent particle flux $\Gamma_{\text {turb }}(\omega)$ in L-mode before the onset of the $\mathrm{D}_{\alpha}$ drop (solid lines) and in $\mathrm{H}$-mode after the $\mathrm{D}_{\alpha}$ drop (dashed lines). The total particle flux $\Gamma$ obtained by integrating $\Gamma_{\text {turb }}(\omega)$ over frequency $\omega=2 \pi f$ for each discharge is shown. 


\section{DISCUSSION}

Phase transition models of the $\mathrm{L}$ to $\mathrm{H}$ transition predict that for conditions marginal for the $\mathrm{L}$ to $\mathrm{H}$ transition, the duration of the transition should lengthen and plasma parameters should vary continuously between the $\mathrm{L}$-mode and $\mathrm{H}$-mode states. A highly radiating zone inside the plasma core just above the divertor $\mathrm{X}$-point (X-point MARFE) has been used to access the marginal transition regime $P_{\text {sep }} \approx P_{\text {thres }}$ to study this prediction. Under these conditions, the duration of the $\mathrm{L}$ to $\mathrm{H}$ transition becomes 50-100 times longer than for fast transitions. However, the evolution of the $E_{\mathrm{r}}$ shear, edge density gradient, $\mathrm{H}$-mode pedestal, and fluctuations is essentially unchanged from fast transitions. The only difference is in the speed with which and degree to which the density fluctuation amplitudes are reduced. In fast transitions, the fluctuation amplitudes are rapidly reduced by a large factor. In contrast, in very slow transitions, the fluctuation amplitudes show only a small reduction on a time scale 50-100 $\times$ longer than the fast transitions. The density fluctuation amplitudes eventually recover to near L-mode levels before the onset of ELMs in both the fast and very slow transitions.

If we postulate that the edge electrostatic fluctuations are pressure gradient driven, then the evolution of the fluctuation amplitudes is easily understood. The absolute fluctuation levels in the established ELM-free $\mathrm{H}$-mode are at or near L-mode levels in the presence of a 4-5 times larger pressure gradient drive, which is evidence of suppression. The lack of a rapid, large reduction at the onset of the recycling drop in the very slow $\mathrm{L}$ to $\mathrm{H}$ transitions also follows from the realization that in fast transitions the fluctuations are suppressed within $100 \mu$ s [7] which is much shorter than an edge confinement time $\tau_{\text {edge }}$ of several milliseconds. Since $\tau_{\text {edge }}$ sets the time scale on which the pressure gradient can change, there is initially a rapid reduction in fluctuation amplitude, followed by a slow increase as the edge pressure gradient responds to the reduced turbulent transport levels. In very slow transitions, the time scales for flucuation suppression and pressure gradient increase are comparable, and the absolute fluctuation amplitudes remain unchanged or increase a small amount. 


\section{REFERENCES}

[1] Burrell, K.H., Plasma Phys. Control. Fusion 36 (1994) A291.

[2] Burrell, K.H., Phys. Plasmas 4 (1997) 1499.

[3] Diamond, P.H., et al., Phys. Rev. Lett. 72 (1994) 2565.

[4] Carreras, B.A., et al., Phys. Plasmas 1 (1994) 4014.

[5] Carreras, B.A., et al., Phys. Plasmas 2 (1995) 2744.

[6] Charlton, L.A., et al., Phys. Plasmas 1 (1994) 2700.

[7] Moyer, R.A., et al., Phys. Plasmas 2 (1995) 2397.

[8] Zohm, H., Phys. Rev. Lett. 72 (1994) 222.

[9] Zohm, H., et al., Plasma Phys. Control. Fusion 37 (1995) 437.

[10] Baker, D., et al., Nucl. Fusion 22 (1982) 807.

[11] Lipschultz, B., et al., Nucl. Fusion 24 (1984) 977.

[12] Drake, J.F., Phys. of Fluids 30 (1987) 2429.

[13] Moyer, R.A., et al., submitted to: Nucl. Fusion (1997)

[14] Gohil, P., et al. in 14th Symp. on Fusion Engineering. 1992: IEEE.

[15] Groebner, R.J., Proc. this conference; to be published in: Plasma Phys. Control. Fusion (1997)

[16] Rettig, C.L., et al., Rev. Sci. Instrum. 66 (1995) 848.

[17] Philipona, R., et al., Phys. Fluids B 5 (1993) 87. 


\section{ACKNOWLEDGMENT}

This work supported by U.S. DOË contracts DE-AC03-89ER51114, W-7405-ENG48, DE-AC05-96OR22464, DE-AC04-94AL85000 and grants DE-FG03-95ER54294 and DE-FG03-85ER53266. The assistance of the DIII-D Team and Operations Group in operating the tokamak and supporting diagnostics is gratefully acknowledged. 


\section{M98004684}

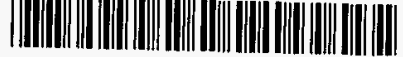

Report Number (14) $Q A--A 22726$ CONF-9709100 -

Publ. Date (11)

199709

Sponsor Code (18) $\frac{D O E / E R, X F}{U C-42 O, D O E / E R}$
UC Category (19) 